\title{
Posterior Reversible Encephalopathy Syndrome : A Rare Cause of Sudden Onset Vision Loss in Pregnancy
}

\author{
Chaudhary R, Dadhich S, Vyas J \\ Department of Obs/Gyn, S P Medical College and attached group of Hospital, Bikaner (Rajasthan) India
}

\begin{abstract}
Posterior Reversible Encephalopathy Syndrome (PRES) is a very rare cause of sudden onset vision loss in pregnancy. There are only few case reports in literature. It is characterized by headache, seizures, altered mental status and visual deficits ranging from visual neglect to cortical blindness. Here a case of PRES has been reported with discussion on its pathophysiology.
\end{abstract}

Keywords: Encephalopathy, pregnancy, vision loss

\section{INTRODUCTION}

Vision loss in pregnancy is a rare occurrence. Posterior Reversible Encephalopathy Syndrome (PRES) is a very rare cause of sudden vision loss in pregnancy. It is a neuroradiologic diagnosis, characterized by headache, seizures, altered mental status and a spectrum of visual deficits ranging from visual neglect to cortical blindness. ${ }^{1}$ Here a case of 23 years old lady is being reported who presented to us with sudden onset vision loss with Posterior reversible encephalopathy syndrome.

\section{CASE}

A twenty three years old primigravida at 34 weeks of gestation presented to us in emergency department of our hospital. She had chief complaints of sudden loss of vision, holocranial headache, vomiting and altered sensorium for 3 hours. Her past, personal and drug history were not significant. On examination she was drowsy and was obeying commands poorly. Her visual acuity examination revealed no perception of light bilaterally with bilateral sluggishly reactive equal pupils. Her blood pressure was 180/120 mm of $\mathrm{Hg}$ and she had bilateral pedal edema. On fundoscopic examination media was clear in both eyes with fundal glow present. Disc margin were well defined, bilateral peripheral retina appeared normal with no evidence of retinal detachment. On investigations there was 1+ proteinuria. Her hemoglobin was $8.6 \mathrm{gm} \%$ with normal platelet count and normal liver and renal function

\section{CORRESPONDENCE}

Dr. Shaifali Dadhich, MS

Department of Obstetrics and Gynaecology

S P Medical College and attached group of Hospitals

Bikaner 334003 Rajasthan, India

Phone: +91-9214018793

Email: shaifalidr23@gmail.com tests. She was treated on the line of preeclampsia with Inj. Labetalol $20 \mathrm{mg}$ IV and prophylactic Magnesium sulfate to prevent eclampsia.

She had a normal vaginal delivery within four hours of admission which was uneventful. Postnatally MRI scan was performed which demonstrated bilateral hyper-intensities in the occipital and posterior parietal lobes on $\mathrm{T} 2$ weighted image (Figure 1). Rest of the brain was normal. She was diagnosed as posterior reversible encephalopathy syndrome and managed conservatively. Her vision started improving from the first postnatal day and she recovered completely by the third day.

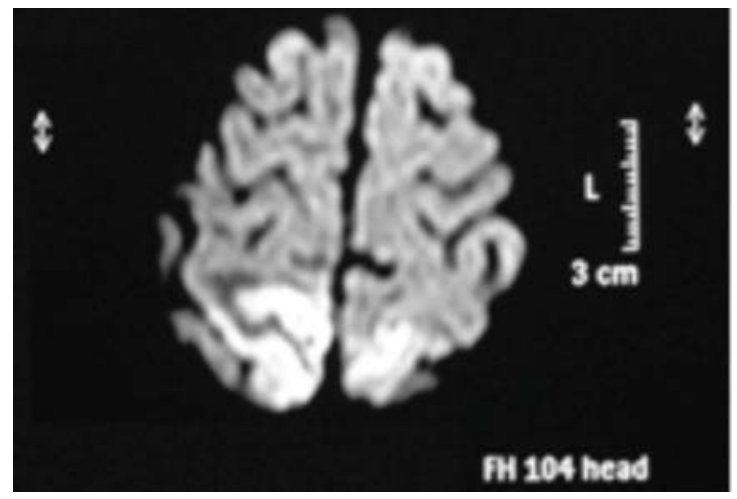

Figure 1. T2 WI MRI images demonstrating symmetric hyper-intensities in bilateral parietal and occipital lobes; characteristic of posterior reversible encephalopathy syndrome 


\section{COMMENT}

Common causes of visual loss in pregnancy are varied and includes thrombotic disorders of eyes and central nervous system, coagulation disorders, hyperemesis gravidarum, eclampsia and preeclampsia, central serous retinopathy and optic neuritis. Posterior reversible encephalopathy syndrome is a very rare cause of sudden vision loss in pregnancy. It is often related to an acute increase in arterial blood pressure and is clinically indistinguishable from hypertensive encephalopathy.

PRES, also called "reversible posterior leukoencephalopathy," typically presents with symptoms of headache, visual changes, seizures, and mental status changes. Visual changes range from loss of acuity, visual neglect, or homonymous hemianopia to complete cortical blindness. PRES is associated with an acute increase in arterial blood pressure, acute renal failure and use of immunosuppressive or cytotoxic drugs (such as methylprednisolone, cyclosporine and tacrolimus). ${ }^{2,3}$

The pathophysiology of PRES is most likely, and most often reported as, vasogenic edema secondary to an acute increase in arterial blood pressure, which overwhelms the autoregulatory capacity of the cerebral vasculature, causing arteriolar vasodilation and endothelial dysfunction leading to interstitial extravasation of fluid. This theory posits that an acute and significant episode of hypertension causes cerebral vasoconstriction with subsequent ischemia and formation of cytotoxic edema. Diffusion-weighted MRI usually indicates vasogenic edema. The posterior circulation is thought to be more susceptible to this type of damage, because there is less sympathetic innervation of the vertebrobasilar vasculature to protect the parenchyma from rapid increases in arterial blood pressure. ${ }^{4,5}$
Radiographically, PRES manifests on CT as hypodensities of the posterior white and gray matter. Lesions are generally bilateral and parieto-occipital, but may involve temporal or frontal lobes, brainstem or cerebellum. 2,6 T2-weighted MRI shows areas of hyper-intense signal and is thought to capture the images with the best quality. ${ }^{5}$

Treatment of arterial blood pressure or withdrawal of the offending drug(s) is the treatment of choice and usually results in complete resolution of the deficits over several days to several weeks, although partial resolution has been reported and the disease can be fatal. PRES, especially in the patient presenting with the typical symptoms of headache, seizures, visual deficits and mental status changes, should be considered and treated without delay to maximize the potential for reversibility.

\section{REFERENCES}

1. Hinchey J, Chaves C, Appignani B, et al. A reversible posterior leukoencephalopathy syndrome. N Eng J Med.1996;334:494-500.

2. Stott VL, Hurrell MA, Anderson TJ. Reversible posterior leukoencephalopathy syndrome: A misnomer reviewed. Intern Med J.2005;35:83-90.

3. Kastrup O, Maschke M, Wanke I, Diener HC. Posterior reversible encephalopathy syndrome due to severe hypercalcemia. J Neurolo.2002;249:1562-6.

4. Vaughan CJ, Delanty N. Hypertensive emergencies. Lancet. 2000; 356:411-17.

5. Witlin AG, Friedman SA, Egerman RS, Frangieh AY, Sibai BM. Cerebrovascualr disorders complicating pregnency: beyond eclampsia. Am J obstet Gynecol.1997;176:1139-48.

6. Servillo G, Striano P, et al. Posterior reversible encephalopathy syndrome (PRES) in critically ill obstetric patients. Intensive Care Med.2003;29:23-6. 